\title{
Asistencia urgente del paciente diabético en el área este de la provincia de Valladolid
}

\author{
M. C. HINOJOSA MENA-BERNAL, E. GONZÁLEZ SARMIENTO, J. HINOJOSA \\ MENA-BERNAL ${ }^{1}$, J. ZURRO HERNÁNDEZ ${ }^{2}$ \\ Departamentos de Medicina Interna y de ${ }^{1}$ Endocrinología. Hospital Clínico \\ Universitario. Valladolid. ${ }^{2}$ Servicio de Urgencias. Hospital Clínico San Carlos. Madrid
}

DIABETIC EMERGENCIES AT EAST AREA OF VALLADOLID

\section{RESUMEN}

Objetivo: Conocer el motivo de asistencia urgente del paciente diabético en el área este de la provincia de Valladolid durante todo un año.

Métodos: Estudio retrospectivo y transversal que analiza una muestra de los pacientes atendidos en el Servicio de Urgencias. Analizamos el motivo de asistencia urgente, bien fuera debido a una complicación directa de la diabetes, o a otro motivo no relacionado con ella. Se aplicó el test t para la comparación de medias y el test Chi cuadrado para la comparación de proporciones.

Resultados: Se atendieron un total de $2.433(2,4 \%)$ urgencias de pacientes diabéticos. El 17,1\% correspondían a complicaciones directamente relacionadas con la diabetes, predominando las complicaciones metabólicas, y dentro de estas la descompensación hiperglucémica.

Conclusión: Destacar la gran prevalencia de las complicaciones diabéticas como motivo de asistencia urgente en nuestro hospital.

PALABRAS CLAVE: Diabetes. Urgencias.

\begin{abstract}
Objective: To know the cause of admissions of diabetic patients at emergencies room at east area of Valladolid in a year.

Methods: It is a retrospective and trasversal study that analysed a number of the admission of diabetic patients at emergencies room because a direct complication diabetes mellitus or another different cause. We used $t$ and $\chi^{2}$ as statistic tests, considering 0.05 as significance.

Results: Up to 2,433 (2.4\%) were diabetes mellitus emergencies. $17.1 \%$ because a direct complication, most of them methabolic complications (24.6\% hyperglycaemia).

Conclusion: We emphasize the high prevalence of direct complications as emergencies admissions in our hospital.
\end{abstract}

KEY WORDS: Diabetes. Emergencies.

Hinojosa Mena-Bernal MC, González Sarmiento E, Hinojosa Mena-Bernal J, Zurro Hernández. J. Asistencia urgente del paciente diabético en el área este de la provincia de Valladolid. An Med Interna (Madrid) 2004; 21: 7-11.

\section{INTRODUCCIÓN}

La diabetes mellitus es una enfermedad metabólica de alta prevalencia y elevada morbimortalidad. Supone uno de los principales problemas de salud mundial, con un gran impacto socioeconómico. Existen escasos trabajos, tanto a nivel nacional como regional, de carácter epidemiológico, conociéndose poco sobre la prevalencia de la diabetes y sus complicaciones, la mortalidad y los costes de la misma (1-18).

La diabetes es responsable de una gran demanda asistencial hospitalaria. Según los diferentes estudios, supone el 2$4 \%$ de los ingresos, con un $0,5-1,3 \%$ de estos debido a la dia- betes o alguna de sus complicaciones. El $70 \%$ corresponden a complicaciones crónicas, y dentro de éstas, en primer lugar, a las macrovasculares, que a su vez consumen más del $60 \%$ de los recursos hospitalarios (18-20).

Con el fin de conocer el motivo de asistencia urgente de los pacientes diabéticos en el área este de Valladolid, hemos realizado un estudio sobre una muestra de los registros atendidos en el Servicio de Urgencias durante todo un año.

Estos datos pueden ser de gran valor, permitiéndonos obtener un conocimiento mayor acerca de la magnitud de los problemas de esta enfermedad, así como ser una ayuda en nuestro intento para resolverlos.

Trabajo aceptado: 30 de junio de 2003

Correspondencia: Carmen Hinojosa Mena-Bernal. C/ Colmenares 14-5 ${ }^{\circ}$ izda. 47004 Valladolid. e-mail: hinojosac@teleline.es 


\section{MATERIAL Y MÉTODOS}

Se trata de un estudio retrospectivo y transversal que analiza una muestra de las urgencias de los pacientes diabéticos atendidos en el Servicio de Urgencias del Hospital Universitario de Valladolid durante todo un año. El Hospital Universitario de Valladolid de categoría intermedia (grupo 3 en la clasificación de hospitales del INSALUD, entre 6 y 8 especialidades complejas, con trasplantes), cuenta con 777 camas funcionantes, y atiende al área II de la provincia (zona este), éste área acoge a una población total de 269.124 habitantes.

Los datos de los pacientes diabéticos fueron recogidos de la historia de urgencias, obteniéndose una muestra significativa (de forma aleatoria) de 742 registros sobre el total de 2433 urgencias de pacientes diabéticos atendidos en el Servicio de Urgencias durante todo un año. La información de 5 casos de la muestra se perdió en el procesamiento de datos, por lo que fueron 737 los registros analizados. Ninguno de estos registros obtenidos de la información del Servicio de Urgencias derivaron en ingresos; por lo que se consideran urgencias no ingresadas.

El diagnóstico de los pacientes diabéticos se realizó en función de los criterios diagnósticos de diabetes (National Diabetes Data Group, 1979), que entonces estaban vigentes. (21)

Hemos estudiado el motivo de asistencia urgente, bien sea debido a una complicación directa de su diabetes, o a otro motivo no relacionado con ella. Entendemos como complicación directa de la diabetes aquellas registradas por la CIE-9MC con los siguientes códigos de la CIE-9-MC (19-20,22) (Tabla I):

\begin{tabular}{lc} 
& TABLA I \\
& CIE-9-MC \\
\hline Descompensación hiperglucémica & $250,0-790,6$ \\
Cetoacidosis & $250,1-250,3$ \\
Hiperosmolaridad & 250,2 \\
Neuropatía & $250,6-357,2$ \\
Oftalmopatía & $250,5-362,0-362,01-362,02-366,41$ \\
Vasculopatía & $250,7-440,20-440,21-440,24-443,81-444,21-444,22-444,81-785,4$ \\
periférica & \\
Enfermedad cerebrovascular & $431-434,11-434,91-435,9-436-437,2$ \\
Cardiopatía isquémica & $410-412-413-414,0-414,02-414,8$ \\
Nefropatía & $250,4-581,81-583,81-585$ \\
Dermopatía & $117-682,7-707-707,1$ \\
Hipoglucemia & $250,8-251-251$ \\
\hline
\end{tabular}

\section{ESTUDIO ESTADÍSTICO}

Para el análisis de los datos recogidos ha sido utilizado un ordenador Pentium III, la aplicación Microsoft Access y el paquete estadístico SAS 6.04 para Windows.

Dado el volumen de consultas atendidas en urgencias (cercano a 100.000), se decidió extraer una muestra de los registros de pacientes diabéticos atendidas a lo largo de eso mismo año; 742 casos fueron seleccionados.

\section{Extracción de la muestra}

Dado que las fichas de los pacientes están ordenadas por fecha de entrada en el servicio de urgencias, planteamos un muestreo sistemático para la extracción de la muestra.

Partimos de la lista de registros de pacientes diabéticos que acudieron a urgencias durante $1997(\mathrm{n}=2433)$ y queríamos extraer una muestra de tamaño $n=742$. Para ello, dividimos la lista en $\mathrm{k}=\mathrm{N} / \mathrm{n}$ partes iguales. Cuando $\mathrm{n}$ no divide a $\mathrm{N}$ ( $\mathrm{k}$ no es entero), podemos aplicar dos métodos para asegurar estimadores insesgados:

- Intervalos fraccionarios

- Muestra cíclica

Para el presente trabajo aplicamos el segundo procedimiento, basado en lo siguiente:

Generar un número aleatorio entre 1 y $\mathrm{N}$, sea $\mathrm{r}$. La muestra está formada por los individuos $\mathrm{r}, \mathrm{r}+\mathrm{k}, \mathrm{r}+2 \mathrm{k}, \ldots ., \mathrm{r}+(\mathrm{n}-1) \mathrm{k}$, donde $\mathrm{k}$ ha sido truncado. Si llegado un valor, "nos salimos de la población", empezaremos por el inicio.

Queremos hacer notar que los datos disponibles constituían una muestra de registros de urgencias y no de pacientes. La identificación, a partir de esta muestra, del conjunto de pacientes implica un sesgo, ya que cada paciente puede acudir una o más veces a urgencias, y por lo tanto tener probabilidades desiguales de ser incluidos en una hipotética muestra. Sin embargo, dado que el porcentaje de pacientes que acudieron más de una vez al servicio de urgencias resultó bajo $(10 \%)$, y que era imposible extraer una muestra de pacientes adecuada (no se dispone de los datos de pacientes que acudieron a urgencias ni de estimaciones de las probabilidades de extracción), trabajamos con un grupo de 644 pacientes, extraídos de los 742 registros de urgencias.

Se aplicó el test t para la comparación de medias y el test Chi cuadrado para la comparación de proporciones. Se consideró como nivel de significación 0,05 .

\section{RESULTADOS}

\section{CARACTERÍSTICAS CLÍNICAS DE LOS PACIENTES QUE ACUDIERON AL SERVICIO DE URGENCIAS}

Se atendieron en el Servicio de Urgencias de nuestro hospital un total de 98.617 urgencias anuales que no requirieron ingreso. De éstas, $2.433(2,4 \%)$ correspondían a urgencias de pacientes diabéticos. Se obtuvo una muestra significativa (de forma aleatoria) de 742 fichas (correspondían a 644 pacientes). La edad media fue de 65,0 $\pm 15,2$ años, con un predominio de mujeres $(55,9 \%)$, y mayor número de diabéticos tipo $2(84,2 \%)$. El análisis por tipo de diabetes (Tabla II), muestra diferencias, claramente significativas, en la edad, que, como cabría esperar, es más elevada en los diabéticos tipo $2(68,4 \pm 11,5$ vs 47,0 $\pm 19,5, \mathrm{p}=$ 0,0001).

Dos casos $(0,3 \%)$ de las urgencias tuvieron desenlace fatal (exitus), ambas debidas a enfermedades del sistema circulatorio, y en concreto a episodios de edema agudo de pulmón.

La distribución de la prevalencia de las enfermedades asociadas se refleja en la Tabla III. 
TABLA II

\begin{tabular}{|c|c|c|c|}
\hline \multicolumn{4}{|c|}{ TABLA II } \\
\hline \multicolumn{4}{|c|}{$\begin{array}{l}\text { DESCRIPCIÓN DE LOS PACIENTES DIABÉTICOS DE } \\
\text { URGENCIAS, SEGÚN EL TIPO DE DIABETES }\end{array}$} \\
\hline \multirow[t]{3}{*}{ Variables } & & Urgencias $(n=644)$ & \\
\hline & & Tipo de diabetes & \\
\hline & Tipo 1 (n=102) & Tipo 2 (n=542) & p valor* \\
\hline Edad (años) & & & 0,0001 \\
\hline Media \pm d.e. (Mín-Máx) & $47,0 \pm 19,5(6-85)$ & 68,4 Ú 11,5 (25-96) & \\
\hline $\operatorname{Sexo}(\%)$ & & & 0,02 \\
\hline Hombres & $56(54,9)$ & $228(42,1)$ & \\
\hline Mujeres & $46(45,1)$ & $314(57,9)$ & \\
\hline$N^{0}$ de visitas (\%) & & & ns \\
\hline 1 & $97(95,1)$ & $481(88,7)$ & \\
\hline 2 & $5(4,9)$ & $45(8,3)$ & \\
\hline 30 más & 0 & $16(3,0)$ & \\
\hline
\end{tabular}

*Test t para comparación de medias y Chi cuadrado para comparación de proporciones. la oftalmopatía, la retinopatía no proliferante y la proliferante con un $35,7 \%$ respectivamente, seguidas de la catarata diabética $(28,6 \%)$. En la neuropatía, la polineuropatía diabética $(50,0 \%)$, y en la nefropatía, la insuficiencia renal crónica $(50,0 \%)$.

\section{ASISTENCIA POR OTRAS CAUSAS}

Las consultas de urgencias motivadas por otras causas no relacionadas con la diabetes, representaron el $82,9 \%$. Fueron las más frecuentes las lesiones y envenenamientos $(24,5 \%)$; seguidas de los síntomas, signos y estados mal definidos $(13,4 \%)$, las enfermedades del aparato digestivo $(13,4 \%)$ y las enfermedades del sistema nervioso y órganos de los sentidos (11,3\%) (Fig. 2).

Dentro de las lesiones y envenenamientos destacaron, los traumatismos y fracturas $(78,6 \%)$, efecto adverso a fármacos $(8,6 \%)$ y cuerpos extraños $(8,6 \%)$. En los síntomas, signos y estados mal definidos, el dolor precordial $(35,4 \%)$ y el síncope $(22,0 \%)$, en las enfermedades del aparato digestivo, las enfermedades del esófago, estómago y duodeno $(36,5 \%)$ y la gastroenteritis $(29,2 \%)$ y por último en las enfermedades del sistema nervioso y órganos de los sentidos, la conjuntivitis $(29,0 \%)$.

\section{DISCUSIÓN}

En este estudio analizamos los datos de los pacientes diabéticos que fueron atendidos durante un año en el Servicio de Urgencias del área este de la provincia de Valladolid.

Del total de las urgencias atendidas, el 2,4\% correspondieron a pacientes diabéticos, predominando las mujeres y los diabéticos tipo 2. El 17,1\% acuden por una complicación relacionada con la diabetes. Las complicaciones metabólicas $(46,1 \%)$ ocuparon el primer lugar, a expensas de la descom-

TABLA III patía periférica (8\%) (Fig. 1).

Dentro de la dermopatía destacaron por su frecuencia la celulitis $(52,2 \%)$ y la úlcera crónica de la pierna $(21,7 \%)$; en

DISTRIBUCIÓN DE LA PREVALENCIA DE LAS COMPLICACIONES DIABÉTICAS Y OTRAS ENFERMEDADES ASOCIADAS

\begin{tabular}{|c|c|c|c|c|c|}
\hline & \multicolumn{2}{|c|}{ Tipo 1} & \multicolumn{2}{|c|}{ Tipo 2} & \multirow[t]{2}{*}{$p$ valor } \\
\hline & Frecuencias & Porcentajes & Frecuencias & Porcentajes & \\
\hline \multicolumn{6}{|l|}{ Complicaciones diabéticas } \\
\hline Oftalmopatía & 16 & 15,7 & 38 & 7,0 & 0,004 \\
\hline Cardiopatía isquémica & 9 & 8,8 & 56 & 10,3 & ns \\
\hline Vasculopatía periférica & 4 & 3,9 & 29 & 5,4 & ns \\
\hline Nefropatía & 3 & 2,9 & 14 & 2,6 & ns \\
\hline Enfermedad cerebrovascular & 2 & 2,0 & 41 & 7,6 & ns \\
\hline Neuropatía & 2 & 2,0 & 37 & 6,8 & ns \\
\hline Dermopatía & - & - & - & - & \\
\hline \multicolumn{6}{|l|}{ Otras enfermedades asociadas } \\
\hline Hipertensión arterial & 21 & 20,6 & 221 & 40,8 & 0,001 \\
\hline EPOC & 7 & 6,9 & 28 & 5,2 & ns \\
\hline Dislipemia & 4 & 3,9 & 50 & 9,2 & ns \\
\hline Tabaquismo & 2 & 2,0 & 2 & 0,4 & ns \\
\hline Sin enfermedades asociadas & - & - & 1 & 0,2 & ns \\
\hline
\end{tabular}


TABLA IV

DISTRIBUCIÓN DE LA ASISTENCIA POR COMPLICACIONES DIRECTAS DE LA DIABETES

\begin{tabular}{|c|c|c|c|c|c|c|c|}
\hline & \multicolumn{2}{|c|}{ Total } & \multicolumn{2}{|c|}{ Tipo 1} & \multicolumn{2}{|c|}{ Tipo 2} & \multirow[t]{2}{*}{$p$ valor } \\
\hline & Frecuencias & Porcentajes & Frecuencias & Porcentajes & Frecuencias & Porcentajes & \\
\hline Hipoglucemia & 25 & 20 & 7 & 29,2 & 18 & 17,6 & ns \\
\hline Hiperglucémia & 31 & 24,6 & 7 & 29,2 & 24 & 23,5 & ns \\
\hline Dermopatía & 23 & 18,2 & 4 & 16,7 & 19 & 18,6 & ns \\
\hline Oftalmopatía & 14 & 11,1 & 3 & 12,5 & 11 & 10,8 & ns \\
\hline Nefropatía & 2 & 1,5 & 2 & 8,3 & - & - & ns \\
\hline Vasc. periférica & 10 & 8 & 1 & 4,2 & 9 & 8,8 & ns \\
\hline E. cerebrovascular & 18 & 14,3 & - & - & 18 & 17,6 & ns \\
\hline Neuropatía & 2 & 1,5 & - & - & 2 & 2,0 & ns \\
\hline Hiperosmolaridad & 1 & 0,8 & - & - & 1 & 1,0 & ns \\
\hline Total & 126 & 100,0 & 24 & 100,0 & 102 & 100,0 & \\
\hline
\end{tabular}

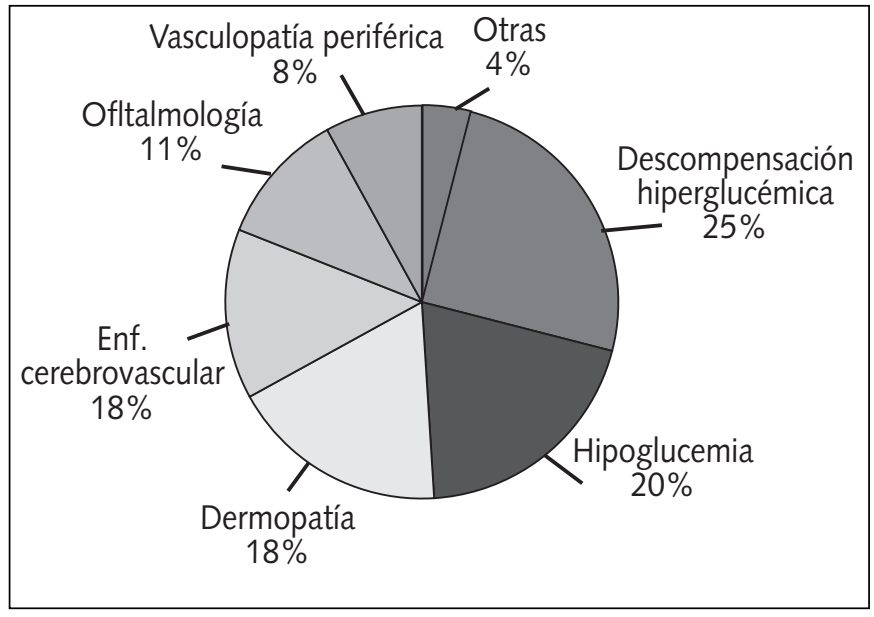

Fig. 1. Distribución de la asistencia por complicaciones directas de la diabetes mellitus.

pensación hiperglucémica $(24,6 \%)$. No hubo ningún caso de cardiopatía isquémica ni de cetoacidosis. Esto puede ser debido a que todos ellos ingresen por su potencial gravedad. Las complicaciones metabólicas fueron más frecuentes en los diabéticos tipo 1, y las vasculares en los tipos 2, aunque sin significación estadística.

La prevalencia de las enfermedades asociadas fue inferior a las descritas por Segado (23). Esto puede deberse a una deficiente cumplimentación de la historia clínica en el Servicio de Urgencias, olvido de diagnóstico de la diabetes y dificultad de atribuirle complicaciones cuando se asocian a otros factores como hipertensión arterial, tabaquismo y obesidad. La inade-

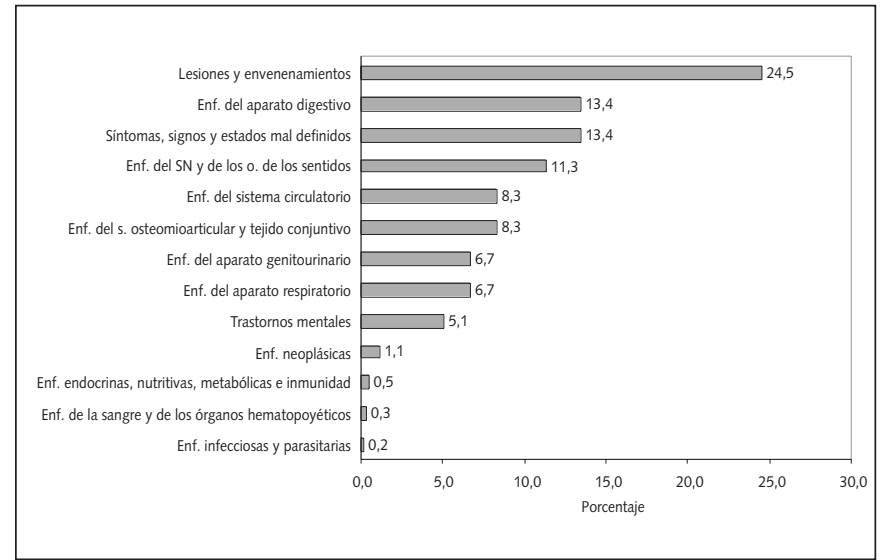

Fig. 2. Distribución de la asistencia por otras causas no relacionadas (CIE-9-MC).

cuada cumplimentación de la ficha de alta, hace que diagnósticos como la obesidad y dislipemia, que se asocian con frecuencia a la diabetes, especialmente tipo 2, tengan una prevalencia inferior que en otros estudios y en especial en el caso del Servicio de Urgencias donde la historia clínica no se recoge de forma minuciosa, aparte, de la dificultad de muchos clínicos a la hora de discernir entre diabetes tipo 1 y tipo 2 .

A pesar de las limitaciones de este estudio, podemos destacar la gran prevalencia de las complicaciones diabéticas, concretamente las metabólicas, y dentro de ellas la descompensación hiperglucémica y la hipoglucemia, como motivo de asistencia en el Servicio de Urgencias debido a una complicación directa de la diabetes, en especial en el diabético tipo 1.

\section{Bibliografía}

1. Hinojosa Mena-Bernal MC, González Sarmiento E, Hinojosa MenaBernal J, Fernández Martínez I, Zurro Hernández J. Estudio epidemiológico de la población diabética ingresada en el hospital universitario de Valladolid en el año 1997. Endocrinol Nutr 2001, 48 (10): 289-294.

2. Hinojosa Mena-Bernal MC, González Sarmiento E, Hinojosa Mena-

Bernal J, Fernández Martínez I, Zurro Hernández J. Prevalencia de los factores de riesgo y de otras enfermedades asociadas en el paciente diabético hospitalizado. Endocrinol Nutr 2002; 49 (5): 136-139.

3. Useros A. Prevalencia de la diabetes mellitus en la provincia de Valladolid. En: Serrano-Ríos M. Epidemiología de la diabetes mellitus: reflexio- 
nes sobre algunos estudios realizados en España y sugerencias para el futuro. Jornadas Internacionales de la Fundación Valgrande 1998, 8: 115-144.

4. Bayo J, Sola C, García F, Latorre PM, Vázquez JA. Prevalencia de la diabetes mellitus no dependiente de la insulina en Lejona (Vizcaya). Med Clin (Barc) 1993; 101: 609-612.

5. Antó Boqué JM, Company Serrat A, Domingo Salvany A, Clos Matheu J. Aproximació a l'epidemiología de la diabetes a ciutat de Barcelona. Gaceta Sanitaria 1985; 19: 11-16.

6. Franch Nadal J, Álvarez Torrices JC. Álvarez Guisasola F, Diego Domínguez F, Hernández Mejía R, Cueto Espinar A. Epidemiología de la diabetes mellitus en la provincia de León. Med Clin (Barc) 1992; 98 : 607-611.

7. Calle-Pascual AL, Vicente A, Martín PJ, Yuste E, Rodríguez C, De Matías J, et al. Estimation of the prevalence of diabetes mellitus diagnosed in Ávila, Spain. Diabetologia 1991; 34 (Supl. 2): 178.

8. Estadísticas de Salud 1978-1987. Información Sanitaria y Epidemiología. Madrid: Ministerio de Sanidad y Consumo, Dirección General de Salud Pública, 1991.

9. Consell Assessor sobre la Diabetes a Catalunya. Estudi de prevalença de diabetes mellitus no insulindependent a Catalunya. Barcelona: III Congreso de la Sociedad Catalana de Diabetes, mayo, 1995.

10. Consenso para la atención a las personas con diabetes en España. Madrid. Ministerio de Sanidad y Consumo, 1994.

11. Anuario Estadístico 1993. Madrid: Instituto Nacional de Estadística, 1994.

12. Monereo Megías S, Fernández San Martín MI, Pedrosa Jamar S, Elviro Peña R. Belmonte Tomas E, Aliaga Maraver A. Prevalencia de la diabetes mellitus registrada en un área sanitaria de la comunidad de Madrid. Endocrinología 1997; 44: 208-212.

13. Goday A. y Serrano-Ríos M. Epidemiología de la diabetes mellitus en España. Revisión crítica y nuevas perspectivas. Med Clin (Barc); 1994, 102, 8: 306-315.
14. Prevención de la diabetes mellitus. Informe de un Grupo de Estudio de la OMS. Organización Mundial de la Salud, Ginebra, serie de Informes Técnicos, núm. 844, 1994.

15. Tamayo M, Faure Nogueras E, Roche Asensio MJ, Rubio Calvo E, Sánchez Ortíz E, Salvador Olivan JA. Prevalence of diabetes and impaired glucose tolerance in Aragón, Spain. Diabetes Care 1997; 20: 534-536.

16. Serna Arnaiz, M.C, Madrid Valls M, Cruz Esteve I, Gasco Eguiluz E, Ribelles M, Serna Majem LI. Estimación de la prevalencia de la diabetes mellitus en seis comarcas de la provincia de Lleida. Endocrinol Nutr 1999; 46: 83-86.

17. Fernández-Vigo J, Sánchez Macho J, Díaz Rey A, Barros J, Tome M, Bueno J. The prevalence of diabetic retinopathy in northwest Spain. An epidemiological study of diabetic retinopathy in Galicia. Acta Ophtalmologica (Copenh) 1993; 71: 22-26.

18. Goicolea I, Mancha AI, Ugarte E, Vázquez JA. Prevalencia de la retinopatía diabética en una población de base hospitalaria. Relación con otras complicaciones diabéticas. Endocrinología 1993; 42, 9: 286-290.

19. Moreneo S, Pavón I, Vega B, Elviro R, Durán M. Complicaciones de la diabetes mellitus: impacto sobre los costes hospitalarios. Endocrinología 1999; 46: 55-59.

20. Jacobs J, Serna M, Fox N. The cost of hospitalization for the late com-

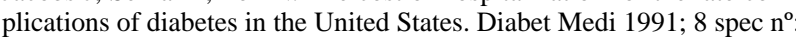
S23-S29.

21. American Diabetes Association. Clinical Practice Recomendations 1992-1993. Diabetes Care 1993; 16 (Supl. 2): 1-118.

22. Aro S, Kangas T, Reunanen A, Salinto M, Koivisto V. Hospital use among diabetic patients and the general population. Diabetes Care 1994; 17: 1320-1329.

23. Segado Soriano A, López González-Cobos C, Granada Martín MJ, Villalba García M, Gil González J, Farfán Sedano A. Patología infecciosa en pacientes diabéticos atendidos en un departamento de urgencias. An Med Interna (Madrid) 1999; 16: 15-21. 